\title{
NILPOTENT CANTOR ACTIONS
}

\author{
STEVEN HURDER AND OLGA LUKINA
}

\begin{abstract}
A nilpotent Cantor action is a minimal equicontinuous action $\Phi: \Gamma \times \mathfrak{X} \rightarrow \mathfrak{X}$ on a Cantor space $\mathfrak{X}$, where $\Gamma$ contains a finitely-generated nilpotent subgroup $\Gamma_{0} \subset \Gamma$ of finite index. In this note, we show that these actions are distinguished among general Cantor actions: any effective action of a finitely generated group on a Cantor space, which is continuously orbit equivalent to a nilpotent Cantor action, must itself be a nilpotent Cantor action. As an application of this result, we obtain new invariants of nilpotent Cantor actions under continuous orbit equivalence.
\end{abstract}

\section{INTRODUCTION}

Let $\Gamma$ be a countably generated group, and let $\Phi: \Gamma \times \mathfrak{X} \rightarrow \mathfrak{X}$, also denoted by $(\mathfrak{X}, \Gamma, \Phi)$, be an action of $\Gamma$ on a topological space $\mathfrak{X}$. We say it is a Cantor action if $\mathfrak{X}$ is a Cantor space.

A nilpotent Cantor action is a minimal equicontinuous Cantor action $(\mathfrak{X}, \Gamma, \Phi)$, where $\Gamma$ contains a finitely-generated nilpotent subgroup $\Gamma_{0} \subset \Gamma$ of finite index. Nilpotent Cantor actions arise in a variety of contexts, which motivates our interest in this class of actions.

A minimal equicontinuous Cantor action is called a generalized odometer in the works [13, 14, 18, 26, and when $\Gamma=\mathbb{Z}$ then $(\mathfrak{X}, \Gamma, \Phi)$ is just a classical odometer, which has been extensively studied [15]. In this work we study properties of generalized odometers given by a virtually nilpotent group action.

A classical odometer is determined up to topological conjugacy by a supernatural number associated to the action (see Bing [7], Aarts and Fokkink [3]). When $\Gamma$ is a finitely-generated free abelian group, then the generalized odometers are classified up to continuous orbit equivalence in the works by Cortez and Medynets [14] and Giordano, Putnam and Skau [18. The nilpotent Cantor actions can be considered as the "simplest" class of Cantor actions whose classification problem is unresolved. One goal of their study is to find invariants of the actions which distinguish them up to topological conjugacy, or better, up to continuous orbit equivalence.

Another motivation for studying nilpotent Cantor actions is that they arise in the classification of renormalizable groups; that is, finitely generated groups which admit a proper self-embedding whose image has finite index [24]. The works by Cornulier [12] and Deré [16 give general criteria for when a nilpotent group admits such a self-embedding. Renormalizable groups arise in a number of geometric and dynamical contexts, such as in the study of laminations with the shape of a compact manifold [1], and in the classification of generalized Hirsch foliations [8.

There is a well-developed theory of the ergodic properties of measure-preserving ergodic actions of nilpotent groups (for example, see the book by Host and Kra [19]), but not so much for the topological dynamics of nilpotent Cantor actions. This paper makes a contribution to their study. The terms in the following result are defined in Section 2

THEOREM 1.1. Let $\left(\mathfrak{X}_{1}, \Gamma_{1}, \Phi_{1}\right)$ be a nilpotent Cantor action which is continuously orbit equivalent to a Cantor action $\left(\mathfrak{X}_{2}, \Gamma_{2}, \Phi_{2}\right)$, then the actions $\Phi_{1}$ and $\Phi_{2}$ are return equivalent. Moreover, if both actions are effective, or faithful, then $\left(\mathfrak{X}_{2}, \Gamma_{2}, \Phi_{2}\right)$ is a nilpotent Cantor action. If both actions are topologically free, then $\Gamma_{1}$ and $\Gamma_{2}$ have nilpotent subgroups of finite index which are isomorphic, and so in particular, $\Gamma_{1}$ and $\Gamma_{2}$ are commensurable.

Version date: November 12, 2020; rev. March 22, 2021.

2010 Mathematics Subject Classification. Primary: 37B05, 37C15, 37C85; Secondary: 57S10.

OL is supported by FWF Project P31950-N35.

Keywords: minimal Cantor actions, topological orbit equivalence, return equivalence, topologically free actions. 
Given Cantor actions $\left(\mathfrak{X}_{1}, \Gamma_{1}, \Phi_{1}\right)$ and $\left(\mathfrak{X}_{2}, \Gamma_{2}, \Phi_{2}\right)$, we can replace them with effective actions by considering the actions of the quotient groups $\Gamma_{1}^{\prime}=\Gamma_{1} / \operatorname{ker}\left(\Phi_{1}\right)$ and $\Gamma_{2}^{\prime}=\Gamma_{2} / \operatorname{ker}\left(\Phi_{2}\right)$ to which Theorem 1.1 applies.

Example 5.2 shows that the conclusion that $\Gamma_{2}$ contains a nilpotent subgroup of finite index is best possible. Example 5.3 shows that if the actions are not topologically free, then the finite-index nilpotent subgroups of $\Gamma_{1}$ and $\Gamma_{2}$ need not be isomorphic, or even commensurable.

Theorem 1.1 suggests that invariants of continuous orbit equivalence for nilpotent Cantor actions must be "virtual" in nature, and depend on properties of nilpotent groups of a special nature. Here is one such invariant.

The virtual nilpotency class $\mathrm{vc}(\Gamma)$ of a finitely-generated virtually nilpotent group $\Gamma$ is defined as the length of a central series for a torsion-free nilpotent subgroup of finite index. This is discussed further in Section 6. The proof of Theorem 1.1 yields the following result.

THEOREM 1.2. Let $\left(\mathfrak{X}_{1}, \Gamma_{1}, \Phi_{1}\right)$ and $\left(\mathfrak{X}_{2}, \Gamma_{2}, \Phi_{2}\right)$ be effective Cantor actions, with $\Gamma_{1}$ and $\Gamma_{2}$ finitely generated. Suppose that $\left(\mathfrak{X}_{1}, \Gamma_{1}, \Phi_{1}\right)$ is a nilpotent action, and $\left(\mathfrak{X}_{2}, \Gamma_{2}, \Phi_{2}\right)$ is continuously orbit equivalent to $\left(\mathfrak{X}_{1}, \Gamma_{1}, \Phi_{1}\right)$. Then $\left(\mathfrak{X}_{2}, \Gamma_{2}, \Phi_{2}\right)$ is a nilpotent Cantor action, and $v c\left(\Gamma_{1}\right)=v c\left(\Gamma_{2}\right)$.

As a second application, in the work 23] the authors study the asymptotic prime spectrum of an equicontinuous Cantor action, which is a generalization of the invariant which classifies equicontinuous actions of $\mathbb{Z}$ as in $[3,7]$. Theorem 1.1 implies that the asymptotic prime spectrum is an invariant of nilpotent Cantor actions under continuous orbit equivalence.

Associated to an equicontinuous Cantor action $(\mathfrak{X}, \Gamma, \Phi)$ is a reduced $C^{*}$-algebra $C_{r}^{*}(\mathfrak{X}, \Gamma, \Phi)$ with a natural choice of Cartan subalgebra, as defined by Renault [28]. Renault studies the properties of Cartan subalgebras and their relation to dynamical systems. The results of [28 and the structure theory for $C^{*}$-algebras of Type I, as in Arveson [4, can be used to define invariants of nilpotent Cantor actions, which are invariants under continuous orbit equivalence by Theorem 1.1 .

In Section 2 we explain the terminology and recall necessary preliminary results for the proof of Theorem 1.1. In Section 3 we show that equicontinuity is preserved by continuous orbit equivalence, and in Section 4 we give a result showing that then the Cantor actions become return equivalent. The proof of Theorem 1.1 is then given in Section 5. The virtual nilpotent class of a virtually nilpotent group and nilpotent Cantor action are defined in Section 6, where we give a proof of Theorem 1.2.

\section{CAntor aCtions}

We recall some of the basic properties of Cantor actions. References for the results described below are the text by Auslander [5], the papers by Cortez and Petite [13, Cortez and Medynets [14, and the authors' works [17] and [22, Section 3].

2.1. Basic concepts. Let $(\mathfrak{X}, \Gamma, \Phi)$ denote an action $\Phi: \Gamma \times \mathfrak{X} \rightarrow \mathfrak{X}$. We write $g \cdot x$ for $\Phi(g)(x)$ when appropriate. The orbit of $x \in \mathfrak{X}$ is the subset $\mathcal{O}(x)=\{g \cdot x \mid g \in \Gamma\}$. The action is minimal if for all $x \in \mathfrak{X}$, its orbit $\mathcal{O}(x)$ is dense in $\mathfrak{X}$.

Let $N(\Phi) \subset \Gamma$ denote the kernel of the action homomorphism $\Phi: \Gamma \rightarrow \operatorname{Homeo}(\mathfrak{X})$. The action is said to be effective if $N(\Phi)$ is the trivial group. That is, the homomorphism $\Phi$ is faithful, and one also says that the action is faithful.

An action $(\mathfrak{X}, \Gamma, \Phi)$ is equicontinuous with respect to a metric $d_{\mathfrak{X}}$ on $\mathfrak{X}$, if for all $\varepsilon>0$ there exists $\delta>0$, such that for all $x, y \in \mathfrak{X}$ and $g \in \Gamma$ we have that $d_{\mathfrak{X}}(x, y)<\delta$ implies $d_{\mathfrak{X}}(g \cdot x, g \cdot y)<\varepsilon$. The property of being equicontinuous is independent of the choice of the metric on $\mathfrak{X}$, compatible with the topology of $\mathfrak{X}$. 
Now assume that $\mathfrak{X}$ is a Cantor space. Let $\mathrm{CO}(\mathfrak{X})$ denote the collection of all clopen (closed and open) subsets of $\mathfrak{X}$, which forms a basis for the topology of $\mathfrak{X}$. For $\phi \in \operatorname{Homeo}(\mathfrak{X})$ and $U \in \operatorname{CO}(\mathfrak{X})$, the image $\phi(U) \in \mathrm{CO}(\mathfrak{X})$. The following result is folklore, and a proof is given in [21, Proposition 3.1].

PROPOSITION 2.1. For $\mathfrak{X}$ a Cantor space, a minimal action $\Phi: \Gamma \times \mathfrak{X} \rightarrow \mathfrak{X}$ is equicontinuous if and only if the $\Gamma$-orbit of every $U \in \mathrm{CO}(\mathfrak{X})$ is finite for the induced action $\Phi_{*}: \Gamma \times \mathrm{CO}(\mathfrak{X}) \rightarrow \mathrm{CO}(\mathfrak{X})$.

We say that $U \subset \mathfrak{X}$ is adapted to the action $(\mathfrak{X}, \Gamma, \Phi)$ if $U$ is a non-empty clopen subset, and for any $g \in \Gamma$, if $\Phi(g)(U) \cap U \neq \emptyset$ implies that $\Phi(g)(U)=U$. Given $x \in \mathfrak{X}$ and clopen set $x \in W$, there is an adapted clopen set $U$ with $x \in U \subset W$. (For a proof of this, see [21, Proposition 3.1].) It follows that the adapted sets containing a point $x \in \mathfrak{X}$ form a local base for the topology. We single out a choice of a base with the following definition:

DEFINITION 2.2. Let $(\mathfrak{X}, \Gamma, \Phi)$ be a Cantor action. A properly descending chain of clopen sets $\mathcal{U}=\left\{U_{\ell} \subset \mathfrak{X} \mid \ell \geq 0\right\}$ is said to be an adapted neighborhood basis at $x \in \mathfrak{X}$ for the action $\Phi$, if $x \in U_{\ell+1} \subset U_{\ell}$ for all $\ell \geq 0$ with $\cap_{\ell>0} U_{\ell}=\{x\}$, and each $U_{\ell}$ is adapted to the action $\Phi$.

A key property is that for $U$ adapted, the set of "return times" to $U$,

$$
\Gamma_{U}=\{g \in \Gamma \mid g \cdot U \cap U \neq \emptyset\}
$$

is a subgroup of $\Gamma$, called the stabilizer of $U$. Then for $g, g^{\prime} \in \Gamma$ with $g \cdot U \cap g^{\prime} \cdot U \neq \emptyset$ we have $g^{-1} g^{\prime} \cdot U=U$, hence $g^{-1} g^{\prime} \in \Gamma_{U}$. Thus, the translates $\{g \cdot U \mid g \in \Gamma\}$ form a finite clopen partition of $\mathfrak{X}$, and are in 1-1 correspondence with the quotient space $X_{U}=\Gamma / \Gamma_{U}$. Then $\Gamma$ acts by permutations of the finite set $X_{U}$ and so the stabilizer group $\Gamma_{U} \subset \Gamma$ has finite index. Note that this implies that if $V \subset U$ is a proper inclusion of adapted sets, then the inclusion $\Gamma_{V} \subset \Gamma_{U}$ is also proper.

2.2. Fixed points for Cantor actions. We next consider the structure of the sets of fixed points for a Cantor action $(\mathfrak{X}, \Gamma, \Phi)$.

The action is free if for all $x \in \mathfrak{X}$ and $g \in \Gamma, g \cdot x=x$ implies that $g=e$, the identity of the group. The isotropy group of $x \in \mathfrak{X}$ is the subgroup

$$
\Gamma_{x}=\{g \in \Gamma \mid g \cdot x=x\} .
$$

Let $\operatorname{Fix}(g)=\{x \in \mathfrak{X} \mid g \cdot x=x\}$, and introduce the isotropy set

$$
\operatorname{Iso}(\Phi)=\{x \in \mathfrak{X} \mid \exists g \in \Gamma, g \neq i d, g \cdot x=x\}=\bigcup_{e \neq g \in \Gamma} \operatorname{Fix}(g) .
$$

DEFINITION 2.3. [10, 26, 28] $(\mathfrak{X}, \Gamma, \Phi)$ is said to be topologically free if $\operatorname{Iso}(\Phi)$ is meager in $\mathfrak{X}$.

Note that if $\operatorname{Iso}(\Phi)$ is meager, then $\operatorname{Iso}(\Phi)$ has empty interior. That is, if there exists a non-identity element $g \in \Gamma$ such that $\operatorname{Fix}(g)$ has interior, then the action is not topologically free.

The notion of topologically free actions was introduced by Boyle in his thesis [9, and later used in the works by Boyle and Tomiyama [10] for the study of classification of general Cantor actions, by Renault [28] for the study of the $C^{*}$-algebras associated to Cantor actions, and by Li [26] for proving rigidity properties of Cantor actions.

The notion of a quasi-analytic action, which was introduced in the works of Álvarez López, Candel, and Moreira Galicia 1, 2], is an alternative formulation of the topologically free property which generalizes to group actions where the acting group can be countable or profinite.

DEFINITION 2.4. An action $\Phi: H \times \mathfrak{X} \rightarrow \mathfrak{X}$, where $H$ is a topological group and $\mathfrak{X}$ a Cantor space, is quasi-analytic if for each clopen set $U \subset \mathfrak{X}$, if the action of $g \in H$ satisfies $\Phi(g)(U)=U$ and the restriction $\Phi(g) \mid U$ is the identity map on $U$, then $\Phi(g)$ acts as the identity on $\mathfrak{X}$.

A topologically free action, as in Definition [2.3, is quasi-analytic. That is, the isotropy set (3) has non-empty interior if $(\mathfrak{X}, H, \Phi)$ is not quasi-analytic. Conversely, the Baire Category Theorem implies that a quasi-analytic effective action of a countable group is topologically free [28, Section 3]. 
A local formulation of the quasi-analytic property was introduced in the works [17, 20, and has proved very useful for the study of the dynamical properties of Cantor actions.

DEFINITION 2.5. An action $\Phi: H \times \mathfrak{X} \rightarrow \mathfrak{X}$, where $H$ is a topological group and $\mathfrak{X}$ a Cantor metric space with metric $d_{\mathfrak{X}}$, is locally quasi-analytic if there exists $\varepsilon>0$ such that for any nonempty open set $U \subset \mathfrak{X}$ with $\operatorname{diam}(U)<\varepsilon$, and for any non-empty open subset $V \subset U$, if the action of $g \in H$ satisfies $\Phi(g)(V)=V$ and the restriction $\Phi(g) \mid V$ is the identity map on $V$, then $\Phi(g)$ acts as the identity on $U$.

2.3. Equivalence of Cantor actions. We recall three notions of equivalence of Cantor actions which we use in this work. This first and strongest notion is the following, as used in [14, 21, 26] :

DEFINITION 2.6. Cantor actions $\left(\mathfrak{X}_{1}, \Gamma_{1}, \Phi_{1}\right)$ and $\left(\mathfrak{X}_{2}, \Gamma_{2}, \Phi_{2}\right)$ are said to be isomorphic if there is a homeomorphism $h: \mathfrak{X}_{1} \rightarrow \mathfrak{X}_{2}$ and group isomorphism $\Theta: \Gamma_{1} \rightarrow \Gamma_{2}$ so that

$$
\Phi_{1}(g)=h^{-1} \circ \Phi_{2}(\Theta(g)) \circ h \in \operatorname{Homeo}\left(\mathfrak{X}_{1}\right) \text { for all } g \in \Gamma_{1} \text {. }
$$

The notion of return equivalence for Cantor actions is defined next. This equivalence is weaker than the notion of isomorphism, and is natural when considering the Cantor systems defined by the holonomy actions for matchbox manifolds, as considered in the works [20, 21, 22.

For a minimal equicontinuous Cantor action $(\mathfrak{X}, \Gamma, \Phi)$ and an adapted set $U \subset \mathfrak{X}$, by a small abuse of notation, we use $\Phi_{U}$ to denote both the restricted action $\Phi_{U}: \Gamma_{U} \times U \rightarrow U$ and the induced quotient action $\Phi_{U}: H_{U} \times U \rightarrow U$ for $H_{U}=\Phi\left(\Gamma_{U}\right) \subset \operatorname{Homeo}(U)$. Then $\left(U, H_{U}, \Phi_{U}\right)$ is called the holonomy action for $\Phi$, in analogy with the case where $U$ is a transversal to a matchbox manifold, and $H_{U}$ is the holonomy group for this transversal.

DEFINITION 2.7. Two minimal equicontinuous Cantor actions $\left(\mathfrak{X}_{1}, \Gamma_{1}, \Phi_{1}\right)$ and $\left(\mathfrak{X}_{2}, \Gamma_{2}, \Phi_{2}\right)$ are said to be return equivalent if there exists an adapted set $U \subset \mathfrak{X}_{1}$ for the action $\Phi_{1}$ and an adapted set $V \subset \mathfrak{X}_{2}$ for the action $\Phi_{2}$, such that the restricted actions $\left(U, H_{1, U}, \Phi_{1, U}\right)$ and $\left(V, H_{2, V}, \Phi_{2, V}\right)$ are isomorphic.

The notion of continuous orbit equivalence for Cantor actions was introduced in [9, 10], and plays a fundamental role in various approaches to the classification of these actions [28]. Consider the equivalence relation on $\mathfrak{X}$ defined by an action $(\mathfrak{X}, \Gamma, \Phi)$,

$$
\mathcal{R}(\mathfrak{X}, \Gamma, \Phi) \equiv\{(x, \gamma x)) \mid x \in \mathfrak{X}, \gamma \in \Gamma\} \subset \mathfrak{X} \times \mathfrak{X} .
$$

Given actions $\left(\mathfrak{X}_{1}, \Gamma_{1}, \Phi_{1}\right)$ and $\left(\mathfrak{X}_{2}, \Gamma_{2}, \Phi_{2}\right)$, we say they are orbit equivalent if there exist a bijection $h: \mathfrak{X}_{1} \rightarrow \mathfrak{X}_{2}$ which maps $\mathcal{R}\left(\mathfrak{X}_{1}, \Gamma_{1}, \Phi_{1}\right)$ onto $\mathcal{R}\left(\mathfrak{X}_{2}, \Gamma_{2}, \Phi_{2}\right)$, and similarly for the inverse map $h^{-1}$.

DEFINITION 2.8. Let $\left(\mathfrak{X}_{1}, \Gamma_{1}, \Phi_{1}\right)$ and $\left(\mathfrak{X}_{2}, \Gamma_{2}, \Phi_{2}\right)$ be Cantor actions. A continuous orbit equivalence between the actions is a homeomorphism $h: \mathfrak{X}_{1} \rightarrow \mathfrak{X}_{2}$ which is an orbit equivalence, and there exist continuous functions $\alpha: \Gamma_{1} \times \mathfrak{X}_{1} \rightarrow \Gamma_{2}$ and $\beta: \Gamma_{2} \times \mathfrak{X}_{2} \rightarrow \Gamma_{1}$ such that:

(1) for each $x \in \mathfrak{X}_{1}$ and $\gamma_{1} \in \Gamma_{1}$, there exists an open set $x \in U_{x} \subset \mathfrak{X}_{1}$ such that $\Phi_{2}\left(\alpha\left(\gamma_{1}, x\right)\right) \circ$ $h\left|U_{x}=h \circ \Phi_{1}\left(\gamma_{1}\right)\right| U_{x} ;$

(2) for each $y \in \mathfrak{X}_{2}$ and $\gamma_{2} \in \Gamma_{2}$, there exists an open set $y \in V_{y} \subset \mathfrak{X}_{2}$ such that $\Phi_{1}\left(\beta\left(\gamma_{2}, y\right)\right) \circ$ $h^{-1}\left|V_{y}=h^{-1} \circ \Phi_{2}\left(\gamma_{2}\right)\right| V_{y}$.

The maps $\alpha$ and $\beta$ are not assumed to be cocycles over the respective actions.

REMARK 2.9. Suppose that $\left(\mathfrak{X}_{1}, \Gamma_{1}, \Phi_{1}\right)$ and $\left(\mathfrak{X}_{2}, \Gamma_{2}, \Phi_{2}\right)$ are actions, and let $h: \mathfrak{X}_{1} \rightarrow \mathfrak{X}_{2}$ be a continuous orbit equivalence. Form the conjugate action $\Psi_{2}: \Gamma_{2} \times \mathfrak{X}_{1} \rightarrow \mathfrak{X}_{1}$ where $\Psi_{2}=h^{-1} \circ \Phi_{2} \circ h$. It then follows that the identity map is an orbit equivalence between the actions $\left(\mathfrak{X}_{1}, \Gamma_{1}, \Phi_{1}\right)$ and $\left(\mathfrak{X}_{1}, \Gamma_{2}, \Psi_{2}\right)$. Thus, we can always reduce to the case where $\mathfrak{X}_{1}=\mathfrak{X}_{2}=\mathfrak{X}$ and $h$ is the identity map, and if $\left(\mathfrak{X}, \Gamma_{1}, \Phi_{1}\right)$ is minimal then $\left(\mathfrak{X}, \Gamma_{2}, \Phi_{2}\right)$ is also minimal. 


\section{Equicontinuous aCtions}

We show that equicontinuity is an invariant of continuous orbit equivalence. The conclusion of Proposition 3.1, with the stronger assumption that both actions are free, was stated in Cortez and Medynets [14, Corollary 4.4], as a consequence of Remark 3 in [27, Section 2] that an isomorphism of full groups is realized spatially for Cantor actions. The proof below follows directly from the definition of a continuous orbit equivalence.

PROPOSITION 3.1. Suppose that Cantor actions $\left(\mathfrak{X}_{1}, \Gamma_{1}, \Phi_{1}\right)$ and $\left(\mathfrak{X}_{2}, \Gamma_{2}, \Phi_{2}\right)$ are continuously orbit equivalent. If both $\Gamma_{1}$ and $\Gamma_{2}$ are finitely generated groups, and $\left(\mathfrak{X}_{1}, \Gamma_{1}, \Phi_{1}\right)$ is equicontinuous, then so is $\left(\mathfrak{X}_{2}, \Gamma_{2}, \Phi_{2}\right)$.

Proof. By Remark 2.9, we can assume that the Cantor spaces are the same, so $\mathfrak{X}=\mathfrak{X}_{1}=\mathfrak{X}_{2}$, and the orbit equivalence $h$ is the identity map on $\mathfrak{X}$. Let $d_{\mathfrak{X}}$ be a metric on $\mathfrak{X}$ compatible with the topology. We must show there exists $\varepsilon_{0}>0$ so that for any $0<\varepsilon \leq \varepsilon_{0}$ there exists $\delta>0$ such that for $x, y \in \mathfrak{X}$ with $d_{\mathfrak{X}}(x, y)<\delta$, and for all $h \in \Gamma_{2}$ we have $d_{\mathfrak{X}}\left(\Phi_{2}(h)(x), \Phi_{2}(h)(y)\right)<\varepsilon$. The idea of the proof of this claim is to show that the action $\Phi_{1}$ has a "shadowing property", using an idea from the proof of [14, Theorem 3.3].

We first establish some technical preliminaries. Let $\alpha$ and $\beta$ be the maps in Definition 2.8 for $h$ the identity map. That is, we have continuous maps $\alpha: \Gamma_{1} \times \mathfrak{X} \rightarrow \Gamma_{2}$ and $\beta: \Gamma_{2} \times \mathfrak{X} \rightarrow \Gamma_{1}$ so that for $y \in \mathfrak{X}$ and $g \in \Gamma_{1}$, there exist a clopen set $y \in U_{g, y} \subset \mathfrak{X}$ with

$$
\Phi_{2}(\alpha(g, y))(z)=\Phi_{1}(g)(z) \text { for } z \in U_{g, y},
$$

and for $h \in \Gamma_{2}$, there exists a clopen set $y \in V_{h, y} \subset \mathfrak{X}$ so that

$$
\Phi_{1}(\beta(h, y))(z)=\Phi_{2}(h)(z) \text { for } z \in V_{h, y} .
$$

Let $\Delta\left(\Gamma_{2}\right) \equiv\left\{h_{1}, \ldots, h_{\mu}\right\} \subset \Gamma_{2}$ be a symmetric set of generators for $\Gamma_{2}$. That is, for $h_{i} \in \Delta\left(\Gamma_{2}\right)$, we have $h_{i}^{-1} \in \Delta\left(\Gamma_{2}\right)$ for all $1 \leq i \leq \mu$.

For each $1 \leq j \leq \mu$, we have an open covering of $\mathfrak{X}$ by the sets $\left\{V_{h_{j}, y} \mid y \in \mathfrak{X}\right\}$. As $\mathfrak{X}$ is compact there exists a Lebesgue number $\varepsilon_{j}>0$ for the covering. Then $\varepsilon^{\prime}=\min \left\{\varepsilon_{1}, \ldots, \varepsilon_{\mu}\right\}>0$ is a Lebesgue number for all of these coverings.

Given $x \in \mathfrak{X}$ there exists an adapted neighborhood basis at $x$ for the action $\Phi_{1}$ as in Definition 2.2 It follows that we can choose an adapted set $W \subset \mathfrak{X}$ for the action $\Phi_{1}$ such that for all $g \in \Gamma_{1}$, we have $\operatorname{diam}_{d_{\mathfrak{X}}}\left(\Phi_{1}(g)(W)\right)<\varepsilon^{\prime}$. Then the translates $\mathcal{W}=\left\{\Phi_{1}(g)(W) \mid g \in \Gamma_{1}\right\}$ form a finite covering of $\mathfrak{X}$ by disjoint clopen sets, and so there is a minimum distance separating them,

$$
\varepsilon^{\prime \prime}=\min \left\{\operatorname{dist}_{d_{\mathfrak{X}}}\left(\Phi_{1}(g)(W), \Phi_{1}\left(g^{\prime}\right)(W)\right) \mid \Phi_{1}(g)(W) \neq \Phi_{1}\left(g^{\prime}\right)(W)\right\}>0 .
$$

Then for $0<\lambda<\varepsilon^{\prime \prime}$ and $y \in \Phi_{1}(g)(W)$, the ball of radius $\lambda$ about $y$ satisfies $B_{d_{x}}(y, \lambda) \subset \Phi_{1}(g)(W)$.

Set $\varepsilon_{0}=\min \left\{\varepsilon^{\prime}, \varepsilon^{\prime \prime}\right\}$ and choose $0<\varepsilon<\varepsilon_{0}$. As the action $\left(\mathfrak{X}, \Gamma_{1}, \Phi_{1}\right)$ is equicontinuous, there exists $\delta>0$ such that for all $g \in \Gamma_{1}$ and $x, y \in \mathfrak{X}$ with $d_{X}(x, y)<\delta$, then $d_{\mathfrak{X}}\left(\Phi_{1}(g)(x), \Phi_{1}(g)(y)\right)<\varepsilon$. Note that $\delta \leq \varepsilon$ as we can take $g$ to be the identity element.

By the above choices, we have that for $x \in \mathfrak{X}$ and each $1 \leq j \leq \mu$, there is

- $g_{x} \in \Gamma_{1}$ such that $x \in \Phi_{1}\left(g_{x}\right)(W)$

- $z_{x, j} \in \mathfrak{X}$ so that $B_{d_{\mathfrak{X}}}(x, \varepsilon) \subset \Phi_{1}\left(g_{x}\right)(W) \subset V_{h_{j}, z_{x, j}}$

where the set $V_{h_{j}, z_{x, j}}$ is defined by (7).

Now let $x, y \in \mathfrak{X}$ satisfy $d_{\mathfrak{X}}(x, y)<\delta$, and let $h \in \Gamma_{2}$. We show that $d_{\mathfrak{X}}\left(\Phi_{2}(h)(x), \Phi_{2}(h)(y)\right)<\varepsilon$.

First, express $h$ in terms of the generators $\Delta\left(\Gamma_{2}\right)$, so $h=h_{j_{m}} \cdots h_{j_{1}}$ for indices $1 \leq j_{\ell} \leq \mu$. We proceed by induction on the factors of $h$. Set $x_{0}=x, y_{0}=y$, then recursively define for $0 \leq \ell<m$,

$$
x_{\ell+1}=\Phi_{2}\left(h_{j_{\ell+1}}\right)\left(x_{\ell}\right) \quad, \quad y_{\ell+1}=\Phi_{2}\left(h_{j_{\ell+1}}\right)\left(y_{\ell}\right) .
$$


Let $g_{x, 0} \in \Gamma_{1}$ be such that $x_{0} \in \Phi_{1}\left(g_{x, 0}\right)(W)$, then we also have $y_{0} \in \Phi_{1}\left(g_{x, 0}\right)(W)$ by the choice of $\delta$. Then there exists $z_{0} \in \mathfrak{X}$ such that $B_{d_{\mathfrak{X}}}\left(x_{0}, \varepsilon\right) \subset V_{h_{j_{1}}, z_{0}}$ and so also $y_{0} \in V_{h_{j_{1}}, z_{0}}$. It follows that $\beta\left(h_{j_{1}}, x_{0}\right)=\beta\left(h_{j_{1}}, y_{0}\right) \in \Gamma_{1}$. Set $g_{j_{1}}=\beta\left(h_{j_{1}}, x_{0}\right)$ then by (7) we have

$$
x_{1}=\Phi_{2}\left(h_{j_{1}}\right)\left(x_{0}\right)=\Phi_{1}\left(g_{j_{1}}\right)\left(x_{0}\right) \quad, \quad y_{1}=\Phi_{2}\left(h_{j_{1}}\right)\left(y_{0}\right)=\Phi_{1}\left(g_{j_{1}}\right)\left(y_{0}\right) .
$$

Note that $d_{\mathfrak{X}}\left(x_{1}, y_{1}\right)<\varepsilon$ by the the choice of $\delta$ and the equicontinuity hypothesis for $\Phi_{1}\left(g_{j_{1}}\right)$.

Now let $1 \leq \ell<m$, and assume that $\left\{g_{j_{1}}, g_{j_{2}}, \ldots, g_{j_{\ell}}\right\} \subset \Gamma_{1}$ have been chosen so that for $1 \leq i \leq \ell$ we have $d_{\mathfrak{X}}\left(x_{i}, y_{i}\right)<\varepsilon$ with

$$
x_{i}=\Phi_{2}\left(h_{j_{i}}\right)\left(x_{i-1}\right)=\Phi_{1}\left(g_{j_{i}}\right)\left(x_{i-1}\right) \quad, \quad y_{i}=\Phi_{2}\left(h_{j_{i}}\right)\left(y_{i-1}\right)=\Phi_{1}\left(g_{j_{i}}\right)\left(y_{i-1}\right) .
$$

Then there exists $z_{\ell} \in \mathfrak{X}$ such that $B_{d_{\mathfrak{X}}}\left(x_{\ell}, \varepsilon\right) \subset V_{h_{j_{\ell+1}}, z_{\ell}}$ and so also $y_{\ell} \in V_{h_{j_{\ell+1}}, z_{\ell}}$.

It follows that $\beta\left(h_{j_{\ell+1}}, x_{\ell}\right)=\beta\left(h_{j_{\ell+1}}, y_{\ell}\right) \in \Gamma_{1}$. Then set $g_{j_{\ell+1}}=\beta\left(h_{j_{\ell+1}}, x_{\ell}\right)$ and define $\widehat{g}_{\ell+1} \equiv$ $g_{j_{\ell+1}} \cdot g_{j_{\ell}} \cdots g_{j_{1}}$. Then by (77) and the previous choices, we have

$$
\begin{aligned}
& x_{\ell+1}=\Phi_{2}\left(h_{j_{\ell+1}}\right)\left(x_{\ell}\right)=\Phi_{1}\left(g_{j_{\ell+1}}\right)\left(x_{\ell}\right)=\Phi_{1}\left(\widehat{g}_{\ell+1}\right)\left(x_{0}\right) \\
& y_{\ell+1}=\Phi_{2}\left(h_{j_{\ell+1}}\right)\left(y_{\ell}\right)=\Phi_{1}\left(g_{j_{\ell+1}}\right)\left(y_{\ell}\right)=\Phi_{1}\left(\widehat{g}_{\ell+1}\right)\left(y_{0}\right) .
\end{aligned}
$$

Then $d_{\mathfrak{X}}\left(x_{\ell+1}, y_{\ell+1}\right)<\varepsilon$ by the the choice of $\delta$ and the equicontinuity hypothesis for $\Phi_{1}\left(\widehat{g}_{\ell+1}\right)$. Thus, for $\ell=m$ we obtain the estimate

$$
d_{\mathfrak{X}}\left(\Phi_{2}(h)(x), \Phi_{2}(h)(y)\right)=d_{\mathfrak{X}}\left(\Phi_{1}\left(\widehat{g}_{\mu}\right)\left(x_{0}\right), \Phi_{1}\left(\widehat{g}_{\mu}\right)\left(y_{0}\right)\right)<\varepsilon
$$

as was to be shown.

\section{Return EQuivalence}

In this section, we show that the locally quasi-analytic property of an equicontinuous Cantor action is preserved by continuous orbit equivalence. The strategy of the proof is to first show that the actions are return equivalent, as defined in Definition 2.7. Then Corollary 4.7 deduces the locally quasi-analytic property from return equivalence.

In a previous work 22, the authors showed that the stable property for an equicontinuous action is preserved by continuous orbit equivalence. The stable property implies the locally quasi-analytic property, which yields the conclusion of Theorem 4.1 below for stable actions. However, there exists nilpotent Cantor actions which are not stable [23, so we must prove a variant of Theorem 6.10 in 22 for our purposes. The proofs of the stable and the locally quasi-analytic versions of this result have significant overlaps, so when possible we refer to proofs of the corresponding results in [22].

THEOREM 4.1. Let $\left(\mathfrak{X}_{1}, \Gamma_{1}, \Phi_{1}\right)$ and $\left(\mathfrak{X}_{2}, \Gamma_{2}, \Phi_{2}\right)$ be Cantor actions, with both $\Gamma_{1}$ and $\Gamma_{2}$ finitely generated groups. Suppose that the actions are continuously orbit equivalent, and that $\left(\mathfrak{X}_{2}, \Gamma_{2}, \Phi_{2}\right)$ is equicontinuous and locally quasi-analytic. Then $\left(\mathfrak{X}_{1}, \Gamma_{1}, \Phi_{1}\right)$ is return equivalent to $\left(\mathfrak{X}_{2}, \Gamma_{2}, \Phi_{2}\right)$.

Proof. By Remark 2.9], we can assume that the Cantor spaces are the same, so $\mathfrak{X}=\mathfrak{X}_{1}=\mathfrak{X}_{2}$, and the orbit equivalence $h$ is the identity map on $\mathfrak{X}$. Let $d_{\mathfrak{X}}$ be a metric on $\mathfrak{X}$ compatible with the topology. Let $\alpha: \Gamma_{1} \times \mathfrak{X} \rightarrow \Gamma_{2}$ and $\beta: \Gamma_{2} \times \mathfrak{X} \rightarrow \Gamma_{1}$ satisfy the relations (6) and (7). Note that $\left(\mathfrak{X}_{1}, \Gamma_{1}, \Phi_{1}\right)$ is an equicontinuous action by Proposition 3.1 .

The proof that the actions are return equivalent follows from a sequence of results. We first show in Lemma 4.2 that there exists an adapted set $U \subset \mathfrak{X}$ such that the map $\alpha$ restricted to the action of $\Gamma_{1, U}$ on $U$ satisfies the cocycle identity. We then show in Proposition 4.3 that there exists an adapted set $W \subset U$ such that the cocycle $\alpha$ is a coboundary when restricted to the action of $\Gamma_{1, W}$ on $W$, and thus conclude that it induces a group homomorphism on $\Gamma_{1, W}$. Both of these results have their exact counterparts in the proof of [22, Theorem 6.10], so we only include sufficient details of their proofs to establish the notation required for the proof of the new results, Lemmas 4.4, 4.5 and 4.6, which show that this homomorphism induces a return equivalence of the actions. 
Choose $x_{0} \in \mathfrak{X}$, then as $\Phi_{2}$ is locally quasi-analytic, there exists $V \subset \mathfrak{X}$ adapted to the action $\Phi_{2}$ such that $x_{0} \in V$ and the action of $H_{2, V}=\Phi_{2}\left(\Gamma_{2, V}\right) \subset \operatorname{Homeo}(V)$ on $V$ is topologically free. Thus, there exists a dense subset $\mathcal{Z}_{V} \subset V$ such that the action of $H_{2, V}$ is free when restricted to $\mathcal{Z}_{V}$.

Choose an adapted set $U \subset \mathfrak{X}$ for the action $\Phi_{1}$ with $x_{0} \in U \subset V$.

Let $\Gamma_{1, U} \subset \Gamma_{1}$ be the isotropy group of the action $\Phi_{1}$ on $U$, as defined as in (1).

Let $\alpha_{U}: \Gamma_{1, U} \times U \rightarrow \Gamma_{2}$ denote the restriction of the map $\alpha: \Gamma_{1} \times \mathfrak{X} \rightarrow \Gamma_{2}$. Then for each $g \in \Gamma_{1, U}$ and $y \in U$ we have $\Phi_{1}(g)(y) \in U$. Set $h=\alpha\left(g, x_{0}\right) \in \Gamma_{2}$ so that by (6) we have $\Phi_{2}(h)(y)=\Phi_{1}(g)(y)$.

Then $U \subset V$ implies that $\Phi_{2}(h)(V) \cap V \neq \emptyset$ hence $h \in \Gamma_{2, V}$ and $\Phi_{2, V}(h) \in H_{2, V} \subset \operatorname{Homeo}(V)$.

That is, the restriction of $\alpha$ to $\Gamma_{1, U} \times U$ induces a map $\widehat{\alpha}_{U}=\Phi_{2, V} \circ \alpha: \Gamma_{1, U} \times U \rightarrow H_{2, V}$. The action of $H_{2, V}$ on $V$ is topologically free, so we have:

LEMMA 4.2. $\widehat{\alpha}_{U}: \Gamma_{1, U} \times U \rightarrow H_{2, V}$ satisfies the cocycle identity

$$
\widehat{\alpha}_{U}\left(g \cdot g^{\prime}, y\right)=\widehat{\alpha}_{U}\left(g, \Phi_{1}\left(g^{\prime}\right)(y)\right) \cdot \widehat{\alpha}_{U}\left(g^{\prime}, y\right) \quad \text { for } g, g^{\prime} \in \Gamma_{1, U} \text { and } y \in U .
$$

Proof. This follows as in the proof of [26, Lemma 2.8], or that of [22, Proposition 6.12].

The next result asserts that a properly chosen restriction of the cocycle $\widehat{\alpha}_{U}$ in Lemma 4.2 is a coboundary. The proof uses in an essential way that the group $\Gamma_{1}$ is finitely-generated, as this allows factoring the cocycle $\widehat{\alpha}_{U}$ into actions supported on regions of continuity for the continuous orbit equivalence functions in (6) and (77). The idea of the proof is modeled on that of [14, Theorem 3.3], with the variation that we only assume the action of $H_{2, V}$ is topologically free, and do not assume the action $\left(\mathfrak{X}_{1}, \Gamma_{1}, \Phi_{1}\right)$ is locally quasi-analytic.

PROPOSITION 4.3. Let $\left(\mathfrak{X}_{1}, \Gamma_{1}, \Phi_{1}\right)$ and $\left(\mathfrak{X}_{2}, \Gamma_{2}, \Phi_{2}\right)$ be equicontinuous Cantor actions, with both $\Gamma_{1}$ and $\Gamma_{2}$ finitely generated groups. Suppose that the actions are continuously orbit equivalent, and that $\left(\mathfrak{X}_{2}, \Gamma_{2}, \Phi_{2}\right)$ is locally quasi-analytic. Then there exists an adapted set $W \subset \mathfrak{X}$ for the action $\Phi_{1}$ so that the restricted cocycle $\widehat{\alpha}_{W}: \Gamma_{1, W} \times W \rightarrow H_{2, V}$ is induced by a group homomorphism $\widehat{\theta}_{W}: \Gamma_{1, W} \rightarrow H_{2, V}$. That is, for $g \in \Gamma_{1, W}$ and $x \in W$, we have $\widehat{\alpha}_{W}(g, x)=\widehat{\theta}_{W}(g)$.

Proof. This follows exactly as in the proof of [22, Proposition 6.12].

The next steps in the proof of Theorem 4.1 deviate from that of [22, Theorem 6.10], as we must show that there exists an isomorphism of holonomy actions as in Definition 2.7. This follows from the next three results. Set $H_{1, W}=\Phi_{1_{W}}\left(\Gamma_{1, W}\right) \subset \operatorname{Homeo}(W)$, and $H_{2, V}=\Phi_{2}\left(\Gamma_{2, V}\right) \subset \operatorname{Homeo}(V)$.

LEMMA 4.4. $\widehat{\theta}_{W}$ induces a monomorphism $\theta_{W}: H_{1, W} \rightarrow H_{2, V}$.

Proof. We first show that

$$
\operatorname{ker}\left\{\Phi_{1, W}: \Gamma_{1, W} \rightarrow H_{1, W}\right\} \subset \operatorname{ker}\left\{\widehat{\theta}_{W}: \Gamma_{1, W} \rightarrow H_{2, V}\right\} .
$$

Suppose that $g \in \Gamma_{1, W}$ satisfies $\Phi_{1, W}(g)=\operatorname{Id} \in H_{1, W} \subset \operatorname{Homeo}(W)$. Recall that for $y \in \mathcal{Z}_{V} \cap W$ the action of $H_{2, V}$ is free on the orbit of $y$, and we have that $\Phi_{1, W}(g)(y)=y$. For $h=\widehat{\theta}_{W}(g)$, by the identity (6) we have

$$
h \cdot y=\widehat{\theta}_{W}(g)(y)=\widehat{\alpha}_{W}(g, y) \cdot y=\Phi_{1, W}(g)(y)=y .
$$

Thus, $h \in H_{2, W}$ must be the identity map since $y \in \mathcal{Z}_{V}$, so $\widehat{\theta}_{W}(g)=$ Id. Thus (10) is satisfied, so $\widehat{\theta}_{W}$ induces a well-defined homomorphism $\theta_{W}: H_{1, W} \rightarrow H_{2, V}$.

Suppose that $\theta_{W}(\widehat{g})=\operatorname{Id}$ for $\widehat{g} \in H_{1, W}$, then for $g \in \Gamma_{1, W}$ with $\widehat{g}=\Phi_{1, W}(g)$, using the identity (10) again, we have that $h \in H_{2, W}$ is the identity map, so $\theta_{W}$ is injective. 
LEMMA 4.5. The adapted set $W \subset \mathfrak{X}$ for the action $\Phi_{1}$ is also adapted for the action of $\Phi_{2}$.

Proof. Let $h \in \Gamma_{2}$ be such that $\Phi_{2}(h)(W) \cap W \neq \emptyset$. As $W \subset V$ is a clopen set and $\mathcal{Z}_{V} \subset V$ is dense, the set $\mathcal{Z}_{V} \cap W$ is dense in $W$. As $W \cap \Phi_{2}\left(h^{-1}\right)(W)$ is non-empty and open in $W$, there exists $y \in \mathcal{Z}_{V} \cap W \cap \Phi_{2}\left(h^{-1}\right)(W)$ for which we have $\Phi_{2}(h)(y) \in W$.

Set $g=\beta(h, y) \in \Gamma_{1}$ then by (7) we have $\Phi_{1}(g)(y)=\Phi_{2}(h)(y) \in W$. As $W$ is adapted to the action $\Phi_{1}$ we have $\Phi_{1}(g)(W)=W$ so $g \in \Gamma_{1, W}$.

Now set $h^{\prime}=\widehat{\alpha}_{W}(g, x)=\widehat{\theta}_{W}(g) \in \Gamma_{2, V}$, where $\widehat{\theta}_{W}$ is the map defined in Proposition 4.3. Then $\Phi_{2, V}\left(h^{\prime}\right)(y)=\Phi_{2, V}(h)(y)$ and so $\Phi_{2, V}\left(h^{\prime}\right)=\Phi_{2, V}(h) \in H_{2, V}$ as $y \in \mathcal{Z}_{V}$. That is, $h=\widehat{\theta}_{W}(g)$ and so for all $z \in W$ we have $\Phi_{2, V}(h)(z)=\Phi_{1, W}(g)(z) \in W$.

Thus, $W$ is adapted to the action $\Phi_{2}$ as was to be shown.

LEMMA 4.6. For the adapted set $W$, the map $\widehat{\theta}_{W}$ induces an isomorphism $\theta_{W}: H_{1, W} \rightarrow H_{2, W}$.

Proof. For $g \in \Gamma_{1, W}$ set $\widehat{g}=\Phi_{1, W}(g) \in H_{1, W}$. The proofs of Lemmas 4.4 and 4.5 show that $\theta_{W}(\widehat{g}) \in H_{2, W}$. Given $h \in \Gamma_{2, W}$ and $y \in \mathcal{Z}_{V} \cap W$ set $g=\beta(h, y) \in \Gamma_{1}$. Then $\widehat{\theta}_{W}(g)$ and $\Phi_{2}(h)$ agree on an open neighborhood of $y$ in $W$, hence agree on all of $W$ as $W \subset V$. It follows that $\theta_{W}$ is an isomorphism onto.

We have shown that $W \subset \mathfrak{X}$ is adapted to both actions $\Phi_{1}$ and $\Phi_{2}$, and $\theta_{W}: H_{1, W} \rightarrow H_{2, W} \subset H_{2, V}$ is an isomorphism. This completes the proof of Theorem 4.1 .

COROLLARY 4.7. Let $\left(\mathfrak{X}, \Gamma_{1}, \Phi_{1}\right)$ and $\left(\mathfrak{X}, \Gamma_{2}, \Phi_{2}\right)$ be Cantor actions, with both $\Gamma_{1}$ and $\Gamma_{2}$ finitely generated groups. Suppose that the identity map on $\mathfrak{X}$ is a continuous orbit equivalence, and that $\left(\mathfrak{X}, \Gamma_{2}, \Phi_{2}\right)$ is equicontinuous and locally quasi-analytic. Then $\left(\mathfrak{X}, \Gamma_{1}, \Phi_{1}\right)$ is locally quasi-analytic.

Proof. It follows from Theorem 4.1 that the two actions are return equivalent, for an adapted set $W \subset \mathfrak{X}$. As $\left(\mathfrak{X}, \Gamma_{2}, \Phi_{2}\right)$ is locally quasi-analytic, we can chose $W$ sufficiently small so that the induced action of $H_{2, W}$ on $W$ is topologically free. Then the isomorphic action of $H_{1, W}$ on $W$ is also topologically free, and thus the action of $\Gamma_{1, W}$ on $W$ is quasi-analytic. As $W$ is adapted for the action $\Phi_{1}$, it follows that $\left(\mathfrak{X}, \Gamma_{1}, \Phi_{1}\right)$ is locally quasi-analytic.

\section{Nilpotent ACtions}

In this section, we give the proof of Theorem 1.1, and also give examples to illustrate its conclusions.

Let $\left(\mathfrak{X}_{1}, \Gamma_{1}, \Phi_{1}\right)$ be a nilpotent Cantor action. The group $\Gamma_{1}$ satisfies the Noetherian property [6] for increasing chains of subgroups, so the action is locally quasi-analytic by the following result:

THEOREM 5.1. 21, Theorem 1.6] Let $\Gamma$ be a Noetherian group. Then a minimal equicontinuous Cantor action $(\mathfrak{X}, \Gamma, \Phi)$ is locally quasi-analytic.

Let $\left(\mathfrak{X}_{2}, \Gamma_{2}, \Phi_{2}\right)$ be a Cantor action, and assume that $\Gamma_{2}$ is finitely-generated.

Assume that the actions are continuously orbit equivalent. By Remark 2.9], we can assume that the Cantor spaces are the same, so $\mathfrak{X}=\mathfrak{X}_{1}=\mathfrak{X}_{2}$, and the orbit equivalence $h$ is the identity map.

Then by Proposition 3.1, the action $\left(\mathfrak{X}_{2}, \Gamma_{2}, \Phi_{2}\right)$ is equicontinuous, and by Theorem 4.1, the actions $\left(\mathfrak{X}_{1}, \Gamma_{1}, \Phi_{1}\right)$ and $\left(\mathfrak{X}_{2}, \Gamma_{2}, \Phi_{2}\right)$ are return equivalent. Then by Corollary 4.7 the action $\left(\mathfrak{X}_{2}, \Gamma_{2}, \Phi_{2}\right)$ is locally quasi-analytic. Let $W \subset \mathfrak{X}$ be the clopen set adapted to both actions $\Phi_{1}$ and $\Phi_{2}$, chosen as in the proof of Corollary 4.7 so that both actions restricted to $W$ are quasi-analytic.

Let $\Gamma_{1, W} \subset \Gamma_{1}$ be the isotropy subgroup of $W$ for the action $\Phi_{1}$, with holonomy group $H_{1, W}=$ $\Phi_{1, W}\left(\Gamma_{1, W}\right) \subset \operatorname{Homeo}(U)$. Let $\Gamma_{2, W} \subset \Gamma_{2}$ be the isotropy subgroup of $W$ for the action $\Phi_{2}$, with holonomy group $H_{2, W}=\Phi_{2, W}\left(\Gamma_{2, W}\right) \subset \operatorname{Homeo}(W)$. 
Let $\theta_{W}: H_{1, W} \rightarrow H_{2, W}$ be the isomorphism defined in Lemma 4.6 which implements the orbit equivalence between the two actions. As $\Gamma_{1, W} \subset \Gamma_{1}$ has finite index, there exists a nilpotent subgroup $\Lambda_{1} \subset \Gamma_{1, W}$ of finite index, with $\Lambda_{1}$ finitely generated. Then the image $\Lambda_{2}=\widehat{\theta}_{W}\left(\Lambda_{1}\right) \subset H_{2, W}$ is a finitely-generated nilpotent subgroup of finite index.

Suppose that the action $\Phi_{1}$ is topologically free, then the restriction $\Phi_{1, W}: \Gamma_{1, W} \rightarrow H_{1, W}$ is an isomorphism. Likewise, if $\Phi_{2}$ is topologically free, then the restriction $\Phi_{2, W}: \Gamma_{2, W} \rightarrow H_{2, W}$ is an isomorphism. As $\Lambda_{1} \subset \Gamma_{1, W}$ has finite index, and likewise for $\Lambda_{2} \subset \Gamma_{2, W}$, this shows that the groups $\Gamma_{1, W}$ and $\Gamma_{2, W}$ contain isomorphic nilpotent subgroups of finite index, and thus also $\Gamma_{1}$ and $\Gamma_{2}$. In particular, the groups $\Gamma_{1}$ and $\Gamma_{2}$ are commensurable.

Now, assume that the action $\left(\mathfrak{X}_{2}, \Gamma_{2}, \Phi_{2}\right)$ is effective, that is, the action map $\Phi_{2}: \Gamma_{2} \rightarrow \operatorname{Homeo}(\mathfrak{X})$ is injective. Let $K_{2, W} \subset \Gamma_{2, W}$ be the kernel of the restricted map $\Phi_{2, W}: \Gamma_{2, W} \rightarrow H_{2, W}$, which need not be trivial (see Examples 5.2 and 5.3 below).

Let $X_{2, W}=\Gamma_{2} / \Gamma_{2, W}$ be the finite set of cosets of $\Gamma_{2, W}$, with a transitive left $\Gamma_{2}$ action. The action $\Phi_{2}$ induces a map $\Pi_{2, W}: \Gamma_{2} \rightarrow \operatorname{Perm}\left(X_{2, W}\right)$ to the group of permutations of $X_{2, W}$, and $\Gamma_{2, W}$ is the isotropy subgroup of the identity coset $e_{W} \in X_{2, W}$. Let $C_{2, W}=\operatorname{ker}\left(\Pi_{2, W}\right) \subset \Gamma_{2}$ be the kernel of this representation, so $C_{2, W}$ is a normal subgroup of $\Gamma_{2}$ with finite index.

Choose representatives $\left\{h_{i} \in \Gamma_{2} \mid 1 \leq i \leq \nu\right\}$ of the cosets of $\Gamma_{2} / \Gamma_{2, W}$ and set $W_{i}=h_{i} \cdot W$. Then

$$
\mathfrak{X}=W_{1} \cup W_{2} \cup \cdots \cup W_{\nu} .
$$

For $h \in C_{2, W}$, the action of $\Phi_{2}(h)$ on $\mathfrak{X}$ leaves each clopen set $W_{i}$ invariant, so for $y=h_{i} \cdot z \in W_{i}$ with $z \in W$, we have:

$$
h \cdot y=h \cdot h_{i} \cdot z=h_{i} \cdot\left(h_{i}^{-1} h h_{i}\right) \cdot z
$$

where $h_{i}^{-1} h h_{i} \in C_{2, W}$, as $C_{2, W}$ is normal in $\Gamma_{2}$. For $1 \leq i \leq \nu$, define the conjugate action on $\mathfrak{X}$,

$$
\Phi_{2}^{i}(h)(z)=\Phi_{2}\left(h_{i}\right)^{-1} \Phi_{2}(h) \Phi_{2}\left(h_{i}\right)(z), \quad z \in \mathfrak{X} .
$$

Then for $h \in C_{2, W}$, by (12) the restriction $\Phi_{2}(h): W_{i} \rightarrow W_{i}$ is the identity if and only if

$$
h \in \operatorname{ker}\left\{\Phi_{2}^{i}: C_{2, W} \rightarrow H_{2, W} \subset \operatorname{Homeo}(W)\right\} .
$$

For $h \in C_{2, W}$ which is not the identity, we have by assumption that $\Phi_{2}(h)$ is not the identity map on $\mathfrak{X}$, hence there exists some $1 \leq i \leq \nu$ such that $\Phi_{2}(h): W_{i} \rightarrow W_{i}$ is not the identity, and so $h \notin \operatorname{ker}\left\{\Phi_{2}^{i}: C_{2, W} \rightarrow H_{2, W}\right\}$.

Define a representation $\widehat{\rho}_{2}$ of $C_{2, W}$ into a product of $\nu$ copies of $H_{2, W}$ by setting for $h \in C_{2, W}$,

$$
\widehat{\rho}_{2}: C_{2, W} \rightarrow H_{2, W} \times \cdots \times H_{2, W} \quad, \quad \widehat{\rho}_{2}(h)=\Phi_{2}^{1}(h) \times \cdots \times \Phi_{2}^{\nu}(h)
$$

The kernel of $\widehat{\rho}_{2}$ is trivial by the above arguments and the assumption that the action $\Phi_{2}$ is effective.

Recall that $\Lambda_{2}=\theta_{W}\left(\Lambda_{1}\right) \subset H_{2, W}$ is a finitely-generated nilpotent subgroup of finite index.

For $1 \leq i \leq \nu$, let $\Lambda_{2}^{i}=\left(\Phi_{2}^{i}\right)^{-1}\left(\Lambda_{2}\right)$. Then $\Lambda_{2}^{i}$ is a subgroup of finite index in $C_{2, W}$, and so $\Lambda_{2}^{\prime}=\Lambda_{2}^{1} \cap \cdots \cap \Lambda_{2}^{\nu}$ has finite index in $C_{2, W}$ and thus also in $\Gamma_{2}$. Observe that for each $1 \leq i \leq \nu$, we have $\Phi_{2}^{i}\left(\Lambda_{2}^{\prime}\right) \subset \Lambda_{2}$. Moreover, the homomorphism (13) restricts to an embedding

$$
\widehat{\rho}_{2}: \Lambda_{2}^{\prime} \rightarrow \Lambda_{2} \times \cdots \times \Lambda_{2} \text {. }
$$

Thus, $\widehat{\rho}_{2}$ is an injection of $\Lambda_{2}^{\prime}$ into a product of nilpotent groups, which is again nilpotent, and so $\Lambda_{2}^{\prime}$ is a nilpotent group. Hence, $\Gamma_{2}$ is virtually nilpotent, as was to be shown.

EXAMPLE 5.2. We give an elementary example to show that the conclusion that the groups $\Gamma_{1}$ and $\Gamma_{2}$ are commensurable in Theorem 1.1 is best possible.

Let $\Gamma_{0}$ be a finitely-generated, torsion free, infinite nilpotent group, and $\mathfrak{X}_{0}=\widehat{\Gamma}_{0}$ be the profinite completion of $\Gamma_{0}$. Let $\Phi_{0}: \Gamma_{0} \times \mathfrak{X}_{0} \rightarrow \mathfrak{X}_{0}$ be the action by left multiplication. Then the action $\Phi_{0}$ on $\mathfrak{X}_{0}$ is free. 
Let $Q_{1}=\mathbb{Z} / 2 \mathbb{Z} \times \mathbb{Z} / 2 \mathbb{Z}$ be the product of cyclic groups of order two, and let $Q_{2}=\mathbb{Z} / 4 \mathbb{Z}$ be the cyclic group of order 4 . Let $Y=\{0,1,2,3\}$ be the set with 4 elements. Choose identifications $\tau_{1}: Q_{1} \rightarrow Y$ and $\tau_{2}: Q_{2} \rightarrow Y$, which define actions of $Q_{1}$ and $Q_{2}$ on $Y$.

Let $\mathfrak{X}=\mathfrak{X}_{0} \times Y$ be the product Cantor space. Define $\Gamma_{1}=\Gamma_{0} \times Q_{1}$ with the product action $\Phi_{1}$ on $\mathfrak{X}$. Similarly, let $\Gamma_{2}=\Gamma_{0} \times Q_{2}$ with the product action $\Phi_{2}$ on $\mathfrak{X}$. Both actions are minimal, equicontinuous and free. Moreover, both actions have the same orbits on $\mathfrak{X}$ and the orbit map satisfies the conditions in Definition 2.8. However, $\Gamma_{1}$ and $\Gamma_{2}$ are not isomorphic as their characteristic torsion subgroups are not isomorphic. On the other hand, both contain the subgroup $\Gamma_{0}$ with finite index, so are commensurable.

EXAMPLE 5.3. We give an example to show that the hypothesis that the actions $\Phi_{1}$ and $\Phi_{2}$ are topologically free in Theorem 1.1 is necessary to conclude that $\Gamma_{1}$ and $\Gamma_{2}$ are commensurable.

Let $\Gamma_{0}$ be a finitely-generated, torsion free, infinite nilpotent group, and $\mathfrak{X}_{0}=\widehat{\Gamma}_{0}$ be the profinite completion of $\Gamma_{0}$. Let $\Phi_{0}: \Gamma_{0} \times \mathfrak{X}_{0} \rightarrow \mathfrak{X}_{0}$ be the free action by left multiplication.

Choose a non-trivial finite group $Q_{0}$. List the elements $Q_{0}=\left\{q_{1}, \ldots, q_{k}\right\}$ with $q_{1}$ the identity element, then write $\mathfrak{X}=Q_{0} \times \mathfrak{X}_{0}$ as the union of clopen subsets $W_{i}=\left\{q_{i}\right\} \times \mathfrak{X}_{0}$, so $\mathfrak{X}=W_{1} \cup \cdots \cup W_{k}$. Let $Q_{0}$ act on $\mathfrak{X}$ as the identity on the factor $\mathfrak{X}_{0}$, and by left multiplication on the factor $Q_{0}$, so that it transitively permutes the partition $\left\{W_{1}, \ldots, W_{k}\right\}$ of $\mathfrak{X}$.

We now define two minimal equicontinuous actions $\Phi_{1}$ and $\Phi_{2}$ on $\mathfrak{X}$, where $\Phi_{1}$ is a free action, $\Phi_{2}$ is locally quasi-analytic, and the actions are continuously orbit equivalent, but the groups $\Gamma_{1}$ and $\Gamma_{2}$ are not commensurable.

First, define $\Gamma_{1}=Q_{0} \times \Gamma_{0}$ and the action $\Phi_{1}$ is defined as follows. The action of $Q_{0}$ on $\mathfrak{X}$ is that above, while the $\Phi_{1}$-action of $\Gamma_{0}$ acts as the identity on the set $Q_{0}$ and as the $\Phi_{0}$-action of $\Gamma_{0}$ on $\mathfrak{X}_{0}$. Note that this action is free.

Next, define $\Gamma_{2}=Q_{0} \ltimes \Gamma_{0}$ as the wreath product, namely, let $\Gamma_{0}^{\left|Q_{0}\right|}=\left\{f: Q_{0} \rightarrow \Gamma_{0}\right\}$ be the set of functions, and note that there is a shift action

$$
Q_{0} \times \Gamma_{0}^{\left|Q_{0}\right|} \rightarrow \Gamma_{0}^{\left|Q_{0}\right|}:(q, f) \mapsto f^{q}(*)=f\left(q^{-1}(*)\right) .
$$

Then the wreath product $Q_{0} \ltimes \Gamma_{0}^{\left|Q_{0}\right|}$ is a group with group product

$$
\left(q_{1}, f_{1}\right) \circ\left(q_{2}, f_{2}\right)=\left(q_{1} q_{2}, f_{1} f_{2}^{q_{1}}\right) .
$$

The wreath product $\Gamma_{2}$ acts on $Q_{0} \times \mathfrak{X}_{0}$ by

$$
(q, f) \cdot(s, x)=(q s, f(q s)(x)) .
$$

That is, the action (15) permutes the copies of $\mathfrak{X}_{0}$ in the product $Q_{0} \times \mathfrak{X}_{0}$, while acting on each copy of $\mathfrak{X}_{0}$ independently via an element defined by the function $f$.

We show that the identity map on $\mathfrak{X}$ is a continuous orbit equivalence between the actions $\left(\mathfrak{X}, \Gamma_{1}, \Phi_{1}\right)$ and $\left(\mathfrak{X}, \Gamma_{2}, \Phi_{2}\right)$. For that, we define cocycles $\alpha: \Gamma_{1} \times\left(Q_{0} \times \mathfrak{X}_{0}\right) \rightarrow \Gamma_{2}$ and $\beta: \Gamma_{2} \times\left(Q_{0} \times \mathfrak{X}_{0}\right) \rightarrow \Gamma_{1}$ as in Definition 2.8 .

Let $f_{g}: Q_{0} \rightarrow \Gamma_{0}^{\left|Q_{0}\right|}: q \rightarrow g$ be the constant function, and define the following function, which is independent of the second component, and so it satisfies (1) in Definition 2.8.

$$
\alpha((q, g),(s, x)) \mapsto\left(q, f_{g}\right) .
$$

The function $\alpha$ implements a "diagonal" embedding of $\Gamma_{1} \subset \Gamma_{2}$. A straightforward computation using (15) shows that the orbits of $\left(\mathfrak{X}, \Gamma_{1}, \Phi_{1}\right)$ are contained in the orbits of $\left(\mathfrak{X}, \Gamma_{2}, \Phi_{2}\right)$.

Conversely, the following function is independent of $x$ and so it is constant on the clopen sets $W_{i}$, for $i=1, \ldots, k$,

$$
\beta((q, f)(s, x))=(q, f(q s)) .
$$

Thus $\beta$ satisfies (2) in Definition 2.8, and clearly maps orbits of $\left(\mathfrak{X}, \Gamma_{2}, \Phi_{2}\right)$ to orbits of $\left(\mathfrak{X}, \Gamma_{1}, \Phi_{1}\right)$. Thus $\alpha$ and $\beta$ implement a continuous orbit equivalence between the two actions. 
Set $U=W_{1}$. Then $\Gamma_{1, U}=\{e\} \times \Gamma_{0}$. On the other hand, $\Gamma_{2, U}=\{e\} \times \Gamma_{0}^{\left|Q_{0}\right|} \cong\{e\} \times \oplus_{i=1}^{k} \Gamma_{0}^{i}$, where $\Gamma_{0}^{i}=\Gamma_{0}$. Thus, the groups $\Gamma_{1}$ and $\Gamma_{2}$ are not commensurable.

REMARK 5.4. The idea of Example 5.3 is that while an orbit equivalence between actions fixes their orbits, for locally quasi-analytic actions it does not determine the actions of the isotropy groups of clopen sets. This is seen in the above example where the isotropy groups $\Gamma_{1, U}$ and $\Gamma_{2, U}$ are related, but not isomorphic. This construction admits various generalizations. It should also be compared with the proof of Theorem 1.2 below.

\section{ViRTual NilPotenCy Class}

In this section we introduce a property for finitely-generated virtually nilpotent groups which is used to define an invariant for nilpotent Cantor actions. Let $\Lambda$ be a finitely-generated torsion-free nilpotent group. The nilpotency class $\mathrm{c}(\Lambda)$ is the least integer $k$ such that for the lower central series $\Lambda_{0}=\Lambda, \Lambda_{i+1}=\left[\Lambda, \Lambda_{i}\right]$, we have $\Lambda_{k+1}=\{e\}$. Note that $\mathrm{c}\left(\Lambda^{\prime}\right)=\mathrm{c}(\Lambda)$ for any subgroup of finite index $\Lambda^{\prime} \subset \Lambda$.

LEMMA 6.1. Let $\Lambda$ be a finitely-generated nilpotent group. Then there exists a finitely-generated torsion-free subgroup $\Lambda^{\prime} \subset \Lambda$ of finite index.

Proof. A finitely generated nilpotent group is residually finite, hence there exists a descending chain of finite index normal subgroups $\left\{\Lambda_{\ell} \mid \ell \geq 0\right\}$ where $\Lambda_{0}=\Lambda$ and $\cap_{\ell>0} \Lambda_{\ell}=\{0\}$. Let $\Lambda_{t} \subset \Lambda$ be the maximal subgroup of torsion elements, then $\Lambda_{t}$ is finitely generated, hence is a finite group. Moreover, $\Lambda_{t}$ is normal in $\Lambda$, and $\Lambda_{t}$ contains every element of finite order in $\Lambda$. It follows that there exists $\ell_{0}>0$ such that $\Lambda_{t} \cap \Lambda_{\ell_{0}}=\{0\}$. Then set $\Lambda^{\prime}=\Lambda_{\ell_{0}}$.

Now let $\Gamma$ be a virtually nilpotent group, so there exists a finitely generated nilpotent subgroup $\Lambda \subset \Gamma$ of finite index. Then by Lemma 6.1 there exists a torsion-free subgroup $\Lambda^{\prime} \subset \Lambda$ of finite index, so we can assume without loss of generality that $\Lambda$ is torsion free. Moreover, the value $\mathrm{c}(\Lambda)$ is independent of the choice of such $\Lambda^{\prime}$ by the previous remarks.

DEFINITION 6.2. Let $\Gamma$ be a virtually nilpotent group. The virtual nilpotency class $\operatorname{vc}(\Gamma)=c(\Lambda)$ where $\Lambda \subset \Gamma$ is a torsion-free nilpotent subgroup of finite index.

Observe that $\mathrm{vc}(\Gamma)=0$ implies that $\Gamma$ is a finite group, and $\mathrm{vc}(\Gamma)=1$ implies that $\Gamma$ contains an abelian subgroup of finite index. The discrete Heisenberg group $\mathcal{H}$ has $\operatorname{vc}(\mathcal{H})=2$. In addition, note that there are many torsion-free nilpotent groups $\Lambda$ with $c(\Lambda)=2$ that are not congruent to the Heisenberg group (see [25] for example.)

If $\Gamma_{1}$ and $\Gamma_{2}$ are virtually nilpotent groups which are commensurable, that is, have subgroups of finite index which are isomorphic, then $\mathrm{vc}\left(\Gamma_{1}\right)=\mathrm{vc}\left(\Gamma_{2}\right)$.

The following property of the virtual nilpotency class will be used to prove Theorem 1.2

LEMMA 6.3. Let $\Lambda$ be a finitely-generated nilpotent group, and $k \geq 1$ an arbitrary integer. Then for the product group $\Lambda^{k}=\prod_{i=1}^{k} \Lambda_{i}$ where each $\Lambda_{i}=\Lambda$, we then have $\operatorname{vc}(\Lambda)=\operatorname{vc}\left(\Lambda^{k}\right)$.

Proof. Let $\Lambda_{0} \subset \Lambda$ be a subgroup of finite index with $c\left(\Lambda_{0}\right)=\mathrm{vc}(\Lambda)$. Then

$$
\operatorname{vc}\left(\Lambda^{k}\right) \leq c\left(\Lambda_{0}^{k}\right)=c\left(\Lambda_{0}\right)=\operatorname{vc}(\Lambda) .
$$

Conversely, let $D \subset \Lambda^{k}$ have finite index with $c(D)=\operatorname{vc}\left(\Lambda^{k}\right)$.

Then $\Lambda_{0}=D \cap\left(\Lambda \times \prod_{i=2}^{k}\{e\}\right)$ has finite index in $\Lambda$ and satisfies

$$
\operatorname{vc}(\Lambda) \leq c\left(\Lambda_{0}\right) \leq c(D) \leq \operatorname{vc}\left(\Lambda^{k}\right)
$$

which shows the claim. 
We now give the proof of Theorem 1.2. Let $\left(\mathfrak{X}_{1}, \Gamma_{1}, \Phi_{1}\right)$ and $\left(\mathfrak{X}_{2}, \Gamma_{2}, \Phi_{2}\right)$ be effective Cantor actions, with both $\Gamma_{1}$ and $\Gamma_{2}$ finitely generated groups, and assume that the actions are continuously orbit equivalent. If $\left(\mathfrak{X}_{1}, \Gamma_{1}, \Phi_{1}\right)$ is a nilpotent Cantor action, then by Theorem 1.1, the action $\left(\mathfrak{X}_{2}, \Gamma_{2}, \Phi_{2}\right)$ is return equivalent to $\left(\mathfrak{X}_{1}, \Gamma_{1}, \Phi_{1}\right)$. We do not assume that the action are topologically free, therefore, our statement does not follow directly from the second sentence in Theorem [1.1 The statement which we prove here in Theorem 1.2 is weaker. It proves that the groups $\Gamma_{1}$ and $\Gamma_{2}$ have the same virtual nilpotency class, but this need not imply that they contain isomorphic subgroups.

Without loss of generality we may assume that $\mathfrak{X}_{1}=\mathfrak{X}_{2}=\mathfrak{X}$ and that the identity map is an orbit equivalence. Then by the proof of Theorem 4.1, there exists an adapted set $W \subset \mathfrak{X}$ for both actions, and an isomorphism $\theta_{W}: H_{1, W} \rightarrow H_{2, W}$.

We next proceed as in the proof of Theorem 1.1. Let $k \geq 1$ be the index of $\Gamma_{1, W}$ in $\Gamma_{1}$. As the action $\Phi_{1}$ is effective, we have an injective map $\widehat{\rho}_{1}: C_{1, W} \rightarrow \prod_{i=1}^{k} H_{1, W}$ as in (13). Similarly, as the action $\Phi_{2}$ is effective, we also have an injective map $\widehat{\rho}_{2}: C_{2, W} \rightarrow \prod_{i=1}^{k} H_{2, W}$, with the same index $k$. Indeed, $W$ is adapted to both actions, which implies that the index of $\Gamma_{1, W}$ in $\Gamma_{1}$ equals the index of $\Gamma_{2, W}$ in $\Gamma_{2}$.

Let $\Lambda_{1} \subset C_{1, W} \subset \Gamma_{1, W}$ be a nilpotent subgroup of finite index, and without loss of generality we may assume that $c\left(\Lambda_{1}\right)=\operatorname{vc}\left(\Gamma_{1, W}\right)$. Then the image $\Lambda_{1}^{\prime}=\Phi_{1, W}(\Lambda) \subset H_{1, W}$ satisfies $\operatorname{vc}\left(\Lambda_{1}^{\prime}\right) \leq c\left(\Lambda_{1}\right)$.

On the other hand, as $\widehat{\rho}_{1}$ is injective on $C_{1, W}$, we have

$$
c\left(\Lambda_{1}\right)=c\left(\widehat{\rho}_{1}\left(\Lambda_{1}\right)\right) \leq \mathrm{vc}\left(\prod_{i=1}^{k} H_{1, W}\right)=\mathrm{vc}\left(\prod_{i=1}^{k} \Lambda_{1}^{\prime}\right)=\mathrm{vc}\left(\Lambda_{1}^{\prime}\right) .
$$

Thus, $\operatorname{vc}\left(\Gamma_{1, W}\right)=c\left(\Lambda_{1}\right)=\operatorname{vc}\left(\Lambda_{1}^{\prime}\right)$.

By an analogous argument, we have $\operatorname{vc}\left(\Gamma_{2, W}\right)=c\left(\Lambda_{2}\right)=\operatorname{vc}\left(\Lambda_{2}^{\prime}\right)$. Since $\theta_{W}: \Lambda_{1}^{\prime} \rightarrow \Lambda_{2}^{\prime}$ is an isomorphism, $\operatorname{vc}\left(\Gamma_{1}\right)=\operatorname{vc}\left(\Gamma_{1, W}\right)=\operatorname{vc}\left(\Gamma_{2, W}\right)=\operatorname{vc}\left(\Gamma_{2}\right)$. This shows the claim of Theorem 1.2

\section{REFERENCES}

[1] J. Álvarez López and A. Candel, Equicontinuous foliated spaces, Math. Z., 263:725-774, 2009.

[2] J. Álvarez López and M. Moreira Galicia, Topological Molino's theory, Pacific. J. Math., 280:257-314, 2016.

[3] J.M. Aarts and R.J. Fokkink, The classification of solenoids, Proc. A.M.S, 111 :1161-1163, 1991.

[4] W. Arveson, An invitation to $C^{*}$-algebras, Graduate Texts in Mathematics, No. 39, Springer-Verlag, New York-Heidelberg, 1976.

[5] J. Auslander, Minimal flows and their extensions, North-Holland Mathematics Studies, Vol. 153, NorthHolland Publishing Co., Amsterdam, 1988.

[6] R. Baer, Noethersche Gruppen, Math. Z., 66:269-288, 1956.

[7] R. Bing, A simple closed curve is the only homogeneous bounded plane continuum that contains an arc, Canad. J. Math., 12:209-230, 1960.

[8] A. Biś, S. Hurder, and J. Shive, Hirsch foliations in codimension greater than one, In Foliations 2005, World Scientific Publishing Co. Inc., River Edge, N.J., 2006: 71-108.

[9] M. Boyle, Topological orbit equivalence and factor maps in symbolic dynamics, Ph.D. Thesis, University of Washington, 1983.

[10] M. Boyle and J. Tomiyama, Bounded topological orbit equivalence and $C^{*}$-algebras, J. Math. Soc. Japan, 50:317-329, 1998.

[11] A. Clark, S. Hurder and O. Lukina, Pro-groups and generalizations of a theorem of Bing, Topology Appl., 271, 2020; arXiv:1811.00288.

[12] Y. de Cornulier, Gradings on Lie algebras, systolic growth, and cohopfian properties of nilpotent groups, Bull. Soc. Math. France, 144:693-744, 2016.

[13] M.-I. Cortez and S. Petite, G-odometers and their almost one-to-one extensions, J. London Math. Soc., $78(2): 1-20,2008$.

[14] M.I. Cortez and K. Medynets, Orbit equivalence rigidity of equicontinuous systems, J. Lond. Math. Soc. (2), 94:545-556, 2016.

[15] T. Downarowicz, Survey of odometers and Toeplitz flows, Algebraic and topological dynamics (ed. Kolyada, Manin, Ward), Contemporary Mathematics , Vol. 385, American Math. Soc., Providence, RI, 2005, pages 7-37.

[16] J. Deré, Gradings on Lie algebras with applications to infra-nilmanifolds, Groups Geom. Dyn., 11:105-120, 2017.

[17] J. Dyer, S. Hurder and O. Lukina, Molino theory for matchbox manifolds, Pacific J. Math., 289:91-151, 2017. 
[18] T. Giordano, I. Putman and C. Skau, $\mathbb{Z}^{d}$-odometers and cohomology, Groups Geom. Dyn., 13:909-938, 2019.

[19] B. Host and B. Kra, Nilpotent structures in ergodic theory, Mathematical Surveys and Monographs, Vol. 236, American Mathematical Society, Providence, RI, 2018.

[20] S. Hurder and O. Lukina, Wild solenoids, Transactions A.M.S., 371:4493-4533, 2019; arXiv:1702.03032.

[21] S. Hurder and O. Lukina, Orbit equivalence and classification of weak solenoids, Indiana Univ. Math. J., 69:2339-2363, 2020; arXiv:1803.02098.

[22] S. Hurder and O. Lukina, Limit group invariants for non-free Cantor actions, Ergodic Theory Dynam. Systems, to appear; arXiv:1904.11072.

[23] S. Hurder and O. Lukina, The prime spectrum of solenoidal manifolds, submitted, 2020; arXiv:2103.06825.

[24] S. Hurder, O. Lukina and W. van Limbeek, Cantor dynamics of renormalizable groups, submitted; arXiv:2002.01565.

[25] H. Lee and K.-B. Lee, Expanding maps on 2-step infra-nilmanifolds, Topology Appl., 117:45-58, 2002.

[26] X. Li, Continuous orbit equivalence rigidity, Ergodic Theory Dynam. Systems, 38:1543-1563, 2018.

[27] K. Medynets, Reconstruction of orbits of Cantor systems from full groups, Bull. Lond. Math. Soc., 43:11041110, 2011.

[28] J. Renault, Cartan subalgebras in $C^{*}$-algebras, Irish Math. Soc. Bull., 61:29-63, 2008.

Steven Hurder, Department of Mathematics, University of Illinois at Chicago, 322 SEO (m/c 249 ), 851 S. Morgan Street, Chicago, IL 60607-7045

Email address: hurder@uic.edu

Olga Lukina, Faculty of Mathematics, University of Vienna, Oskar-Morgenstern-Platz 1, 1090 Vienna, Austria

Email address: olga.lukina@univie.ac.at 